\title{
Insights into Three-Dimensional Radiofrequency Circuits Connections
}

Rabah Dahmani ${ }^{1}$, Olivier Valorge ${ }^{2}$, Fengyuan Sun $^{1}$, Samir Labiod ${ }^{3}$, Francis Calmon ${ }^{1}$, Saïda Latreche ${ }^{3}$, Ian $\mathrm{O}^{\prime}$ Connor $^{1}$ and Christian Gontrand ${ }^{1}$

1. INL, CNRS-UMR5270, INSA-Lyon, Université de Lyon, Villeurbanne F-69621, France

2. $R^{3}$ Logic, 50 chemin de l'Etoile, Monbonnot-Saint-Martin 38330, France

3. Laboratoire Hyperfréquence \& Semi-conducteur, Département d'Electronique Faculté des Sciences de l'Ingénieur, Université

Mentouri, Constantine 25000, Algeria

Received: April 19, 2011 / Accepted: May 19, 2011 / Published: June 25, 2011.

\begin{abstract}
The authors state briefly the possibility of various simulators to handle propagation of electromagnetic waves along some interconnections, in 3D RF (Radio Frequency) circuits. The studies are first derived in the time domain: a Finite-Difference Time-Domain method is applied, taking spectra via FFTs (Fast Fourier Transform) as post-processors. Electric and magnetic field distributions, pulse propagations along stripline structures or vias are highlighted. The scattering parameters for various cases are extracted and compared. Some original issue of this work is an insight on crosstalk or shielding phenomena between lines.
\end{abstract}

Key words: Maxwell's equations, radio frequency (RF), numerical methods, shielding effects, through silicon via.

\section{Introduction}

Three dimensional integration technologies (3D) allow fabrication of planar devices in vertically stacked planes. Today, the desire of computational accuracy and effectiveness in microwave devices and circuits become an essential part for engineers and designers.

The goal of this paper is twofold: First of all, we develop a Finite Difference Time domain (FDTD) method to resolve the two equations of Maxwell relative to the curl fields - the electric field E, and the magnetic excitation $\mathrm{H}$, starting from the pioneering paper of Yee [1]; As an example support, we consider a simple conductor plate. Then we do a relatively in-depth study of another simple case: a strip line. For that, we use essentially Sentaurus [2-3]; Sentaurus is a software package based on the finite element method (FEM) for the device study and the FDTD one for the

Corresponding author: Christian Gontrand, professor, research fields: telecommunications, noise, 3D integration. E-mail: christian.gontrand@insa-lyon.fr. electromagnetic field.

The principal aim of such a way is to grasp sufficient numerical experiences (plus actual experiments), to pursue the development of our "open" tool (our final goal will be to build a simulator which couples the electromagnetic equations with device equations (cf. "Poisson" and div J).

The paper is organized as follows: The first section deals with our FDTD method applied to Maxwell's equations relative to the curl fields, considering a stripline; section 2 is a comparison between the most well known simulators; then in section 3, we extend this work to two strip lines with shield plane, to study crosstalk phenomena; in section 4 we concern a $3 \mathrm{D}$ TSV study.

\section{The Finite-Difference Time-Domain Method}

Mawxell's equations relative to the vortical fields, considering media as perfect (Homogeneous, Linear and Isotropic) are 


$$
\begin{gathered}
\nabla \times \vec{E}=-\mu \cdot \frac{\partial \vec{H}}{\partial t} \\
\nabla \times \vec{H}=\varepsilon \cdot \frac{\partial \vec{E}}{\partial t}+\sigma \vec{E}
\end{gathered}
$$

where $\mu$ denotes the permeability, $\varepsilon$ the permittivity, and $\sigma$ the electric conductivity.

Although there are different ways to solve Maxwell' equations numerically, the FDTD is one of the most suitable schemes for the purpose of analyzing 3D structures.

Yees's pioneering approach [1, 4] uses centred differences to approximate space and time derivatives. The region being modelled is represented by two interleaved grids, one containing the edges of the electric field, the other containing the edges of the magnetic one.

Time derivatives are not computed at the same time-frame, but at different, interlaced intervals. Then, each unknown field component depends only on previously-calculated components of the dual kind: two sets of equations are thus recovered, each set being composed of independent equations. This so-called "leapfrog" algorithm makes the set of linear equations easy to handle and solve sequentially:

$$
\begin{gathered}
\frac{H_{y: i+1 / 2}-H_{y}: i-1 / 2}{\Delta x}-\frac{H_{x}: i+1 / 2-H_{x}: i-1 / 2}{\Delta y} \\
=\varepsilon \cdot \frac{E_{z}^{n+1}-E_{z}^{n}}{\Delta t}+\vec{J}_{l e, z}\left(\vec{E}_{z}\right)
\end{gathered}
$$

$\vec{J}_{l e, z}\left(E_{z}\right)$ can be evaluated at the instant $(\mathrm{n}+1 / 2) \cdot \Delta \mathrm{t}$ using a semi-implicit approximation:

$$
\vec{J}_{l e, z}^{n+1 / 2}=\vec{J}_{l e, z}^{n+1 / 2}\left(\frac{1}{2}\left[E_{z}^{n+1}+E_{z}^{n}\right]\right)
$$

A stability criterion rewired is:

$$
\Delta t \leq \frac{1}{c \sqrt{\frac{1}{\Delta x^{2}}+\frac{1}{\Delta y^{2}}+\frac{1}{\Delta z^{2}}}}
$$

where $c$ is the propagation speed in the medium and $\Delta \mathrm{x}$, $\Delta y$ et $\Delta z$ are the cell minimum space in the FDTD grid. Finally, the computation domain is chosen large enough to have possibly two ports far away from any discontinuity.

As a first check, after the implementation of our FDTD algorithm, we consider the diffraction of an incident TM wave (Transverse Electro-magnetic mode; initial amplitude shape: a half sinus) by a perfectly conducting rectangular strip line; some numerical results are shown in Fig. 1: wave amplitude-arbitrary unit-versus calculation domain spatial coordinates - i, j indexes. The space grid size must be such that over one increment the electromagnetic field does not change too much: to have meaningful results, the linear dimension of the grid must be only a fraction of the wave length. We shall assume that the field does not depend on the $\mathrm{z}$ direction. The incident wave is well scattered (as waited) after it encounters the obstacle.

\subsection{The Strip Line}

A microstrip is a two conductors transmission line consisting of a strip over a ground plane; both of them are usually supported by a layer of dielectric material. The field around the strip conductor exists both in the air and in the dielectric; this inhomogeneous dielectric arrangement leads to quasi-TEM (Transverse Electromagnetic mode) behavior and greatly complicates the analysis of microstrip components. By quasi-TEM, we mean that there is always some small, but finite, longitudinal component to the fields on a microstrip line.

2.1.1 Analytical Models for Strip Parameter Extraction-ADS Simulator [5]

ADS simulator is a well known RF SPICE-like simulator. Starting from geometrical data of the problem and dealing with some well known analytical formula (e.g. Fig. 2), it is possible to extract the principal characteristics of the strip.

Then, in our typical example, we get $Z_{0}=73.6 \Omega, C_{0}$ $=0.362 \mathrm{pF}, \mathrm{L}_{0}=1.96 \mathrm{nH} . \mathrm{G}_{0}$ is null, as the dielectric is considered perfect.

We consider also the skin effect, modelled by the following well known equations: 

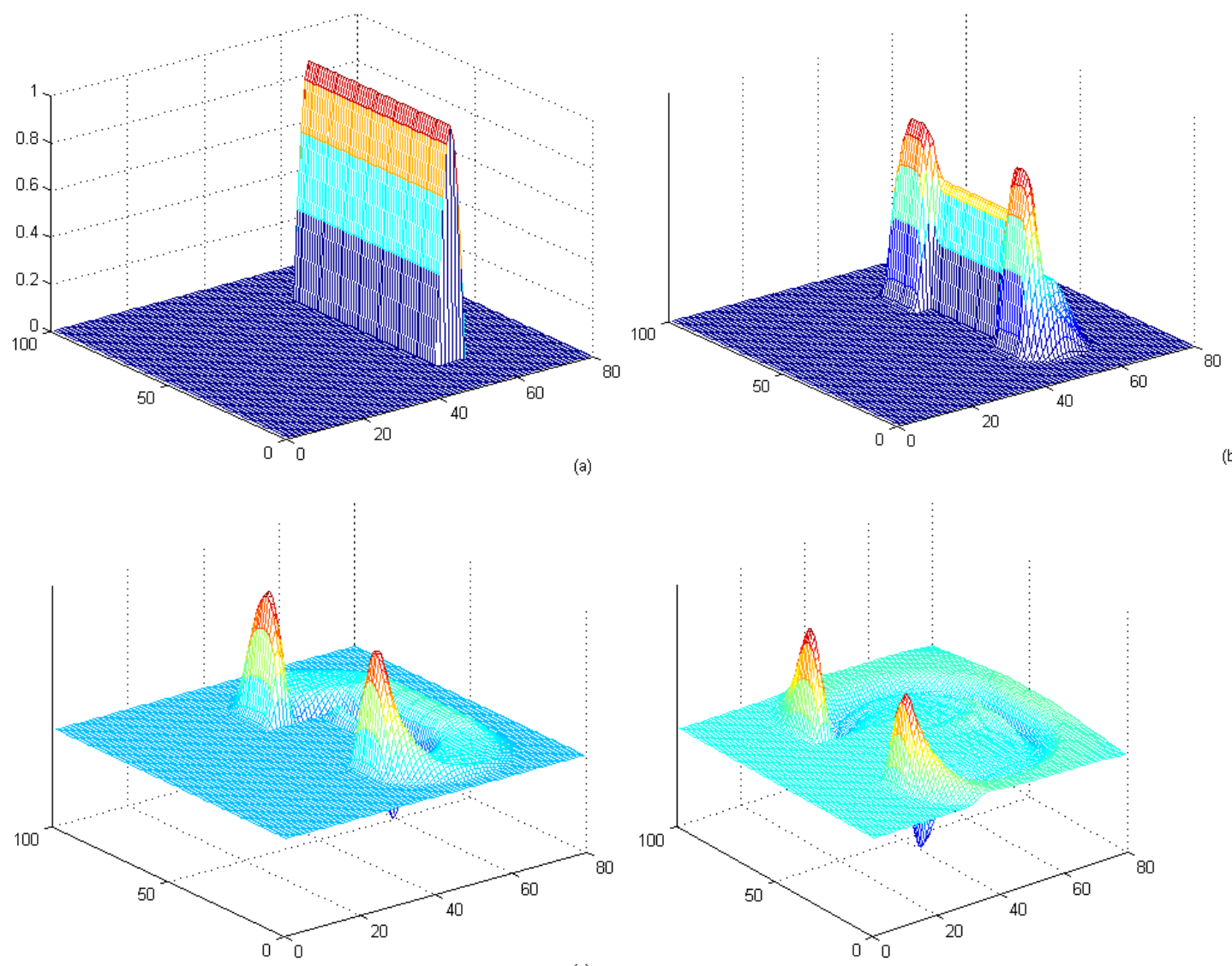

Fig. 1 Half-sinus wave propagating from the right to the left; the obstacle is a perfect conducting rectangle: $i=17$ to $49, j=$ 33 to 65; calculation domain: $\operatorname{imax}=80, j \max =100$.

$$
\begin{array}{ll}
\mathrm{Z}_{0}=\frac{87}{\sqrt{\varepsilon_{\mathrm{r}}+1.41}} \cdot \ln \left(\frac{5.98 \mathrm{H}}{0.8 \mathrm{~W}+\mathrm{T}}\right) & \text { oh m } \\
\mathrm{C}_{0}=\frac{26.4\left(\varepsilon_{\mathrm{r}}+1.41\right)}{\ln \left(\frac{5.98 \mathrm{H}}{0.8 \mathrm{~W}+\mathrm{T}}\right)} \quad \mathrm{F} \cdot \mathrm{m}^{-1}
\end{array}
$$

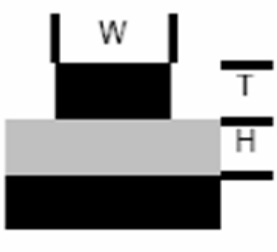

Fig. 2 ADS: analytical relations for capacitance and impedance of a stripline (dielectric: grey color).

$$
\begin{aligned}
& e_{\text {skin }}=\frac{1}{(\mu \cdot \sigma \cdot \pi \cdot f)^{1 / 2}} \\
& \text { if }_{f<f_{\text {skin }}} \\
& R_{0}=\frac{l}{w \cdot t \cdot \sigma} \\
& \text { else } \\
& R_{0}=\frac{l}{w_{\text {skin }} \sigma}
\end{aligned}
$$

\subsubsection{Fasthenry/Fastcap Extraction}

Fasthenry [6] and Fastcap [7] are free tools; with them, we can extract inductance/résistance and capacitance of the $3 \mathrm{D}$ structures.

With the same example as above [5], we get $\mathrm{C}_{0}=$ $0.357 \mathrm{pF}, \mathrm{L}_{0}=2.15 \mathrm{nH}(\mathrm{à} 1 \mathrm{MHz}), \mathrm{R}_{0}=3.18 \mathrm{~m} \Omega$ (à $1 \mathrm{MHz}$ ), and then $\mathrm{Z}_{0}=77.6 \Omega$.

\subsection{Numerical Simulations}

\subsubsection{HFSS and COMSOL Simulations}

HFSS [8] is a dedicated tool for full wave electromagnetic analysis. It uses essentially a Finite Element method (FEM). Typically a 3D simulation can 
use a few hours.

On another hand COMSOL [9], a FEM-based simulator is more general (multiphysics); we could couple EM and device fields.

\subsubsection{Result Comparisons}

To simulate the parameters $\mathrm{S}_{11}$ et $\mathrm{Z}_{11}$ from analytical model or Fastcap/Fasthenry simulations, lumped $\pi$-RLC models are generated with ADS.

For the sake of the basic criterion " $1 / 20$ ", and considering a wave length of $5.9 \mathrm{~mm}$ at $50 \mathrm{GHz}$, the distributed model is constituted of 20 elements (the $\mathrm{R}$, $\mathrm{L}$ and $\mathrm{C}$ values are divided by 20 ).

\subsubsection{Parameters $\mathrm{S}_{11}$}

Presented are on the Figs. 3-4, the imaginary and the

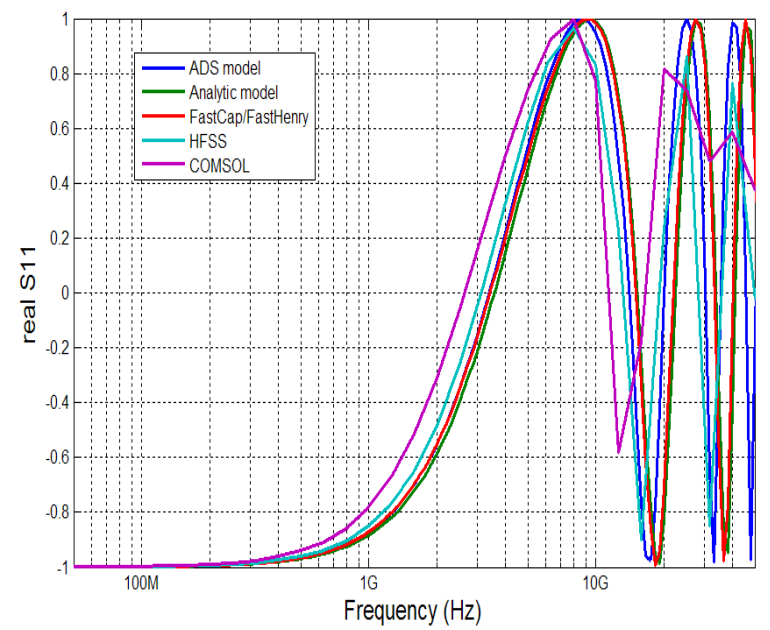

(a)

Fig. 3 (a) Microstrip: short and (b) microstrip: short.

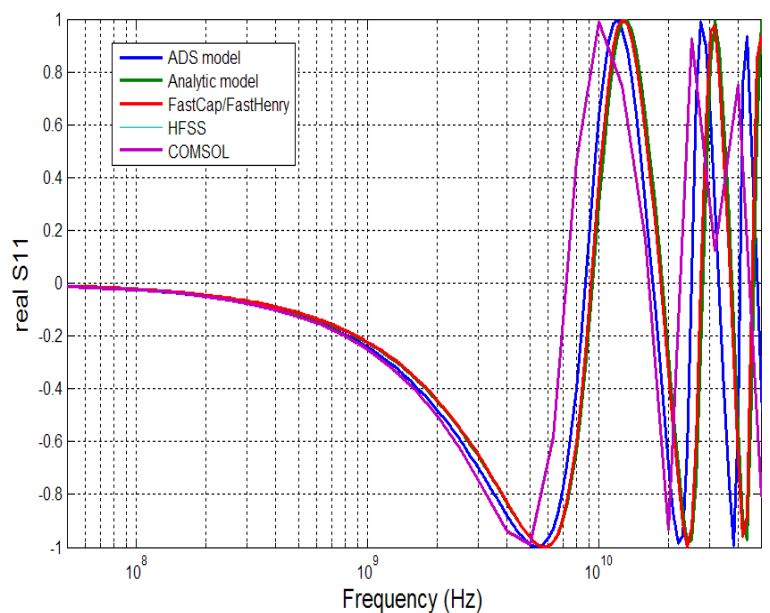

(a) real part of the S-parameters, respectively for a short or open circuit of the microstrip. Some differences appear essentially for the oscillations at high frequencies, depending perhaps on the discretisation scheme, the mesh grid, or the mathematical algorithm.

\subsubsection{Impédance $Z_{11}$}

The $Z_{11}$ impedance is extracted from $S_{11}$ using the following well-known equation:

$$
Z_{11}=\frac{1+S_{11}}{1-S_{11}}
$$

For the sake of clarity, we split the frequency range of wobulation in two ones: $1 \mathrm{MHz}-5 \mathrm{GHz}$ (verifying the criterion of $\lambda / 20$ ) and $5 \mathrm{GHz}-50 \mathrm{GHz}$. We present only the results coming from the first frequency range,

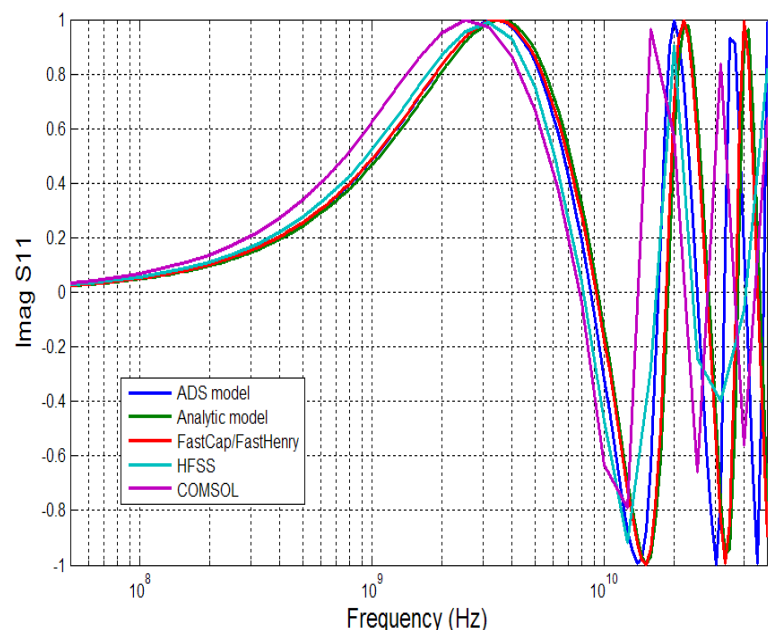

(b)

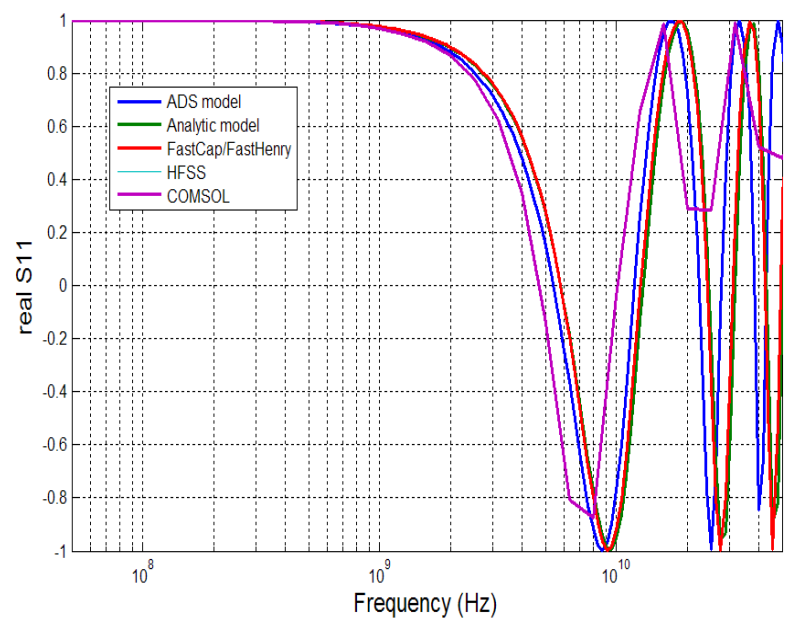

(b)

Fig. 4 (a) Microstrip: open and (b) microstrip: open. 
because we can give some physical interpretation easier, building models with inductances et resistances in series, but also some parallel capacitances. This impedance is presented in Fig. 5; we recall the skin depth varying, in the analytical theory, as the square root of the frequency.

\subsection{Microstrip Open (Parallel Impedance)}

2.3.1 RLC Network and Characteristic Impedance

$\mathrm{C}, \mathrm{L}$ and $\mathrm{R}$ are extracted from "open" simulations (for low frequency), using the following formula:

$$
\begin{gathered}
C_{\text {freq }}=-\frac{1}{\operatorname{im}\left(Z_{11 \text { OPEN }}\right) \cdot 2 \cdot \pi \cdot \text { freq }} \\
L_{\text {freq }}=\frac{i m\left(Z_{11 \text { SHORT }}\right)}{2 \cdot \pi \cdot \text { freq }} \\
R_{\text {freq }}=r e\left(Z_{11 \text { SHORT }}\right)
\end{gathered}
$$

Figs. $5 \mathrm{~b}$ and 6 are depicted the $\mathrm{R}, \mathrm{L}, \mathrm{C}$ results, extracted from the $Z_{11}$ impedance. It is remarked the difference, at low frequencies, for the real part of $Z_{11}$, between HFSS and the others simulations, that is not easy to understand; HFSS does not seems take into account the serie resistance.

Finally, Table 1 presents a result synopsis of RLC values extracted with the dedicated simulators. Again (Fig. 5a) HFSS results deviate a little bit from the other ones.

\subsubsection{Simulations with an FDTD Algorithm}

As we said, Sentaurus-EMW [2]) is a simulator based on the 2D or 3D FDTD algorithm. This tool's interest is twofold: (1) the transient time studies seem of more physical outstanding than the frequency one; in fact, we used FFT algorithm as a postprocessor of the FDTD. (2) Sentaurus is dedicated to the device2D or 3D-(Sentaurus-DESSIS); it should be very useful for 3D studies of RF heterogeneous circuits with substrate coupling and EMC. This simulator seems pretty hard to handle, but it is interesting in a physical point of view.

\subsubsection{The Stripline}

We consider again the basic connecting elements: the striplines [10].

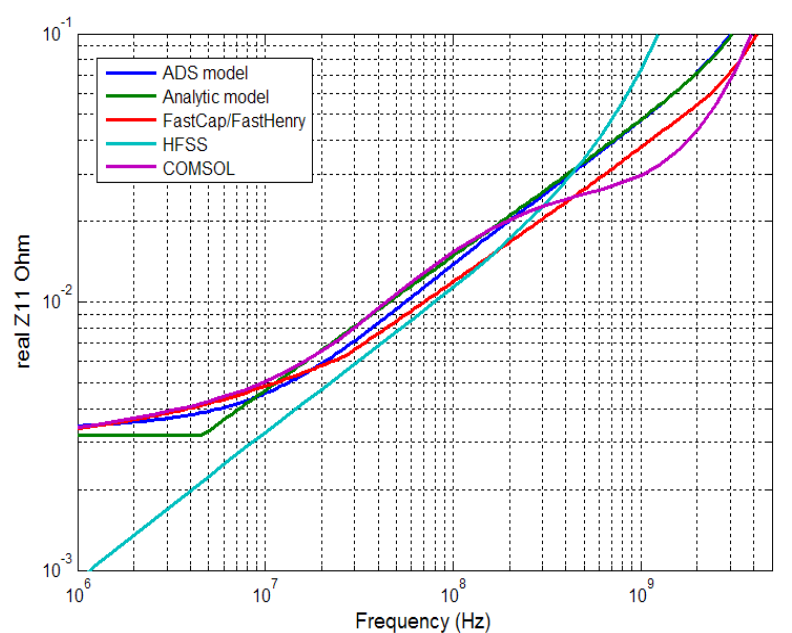

(a)

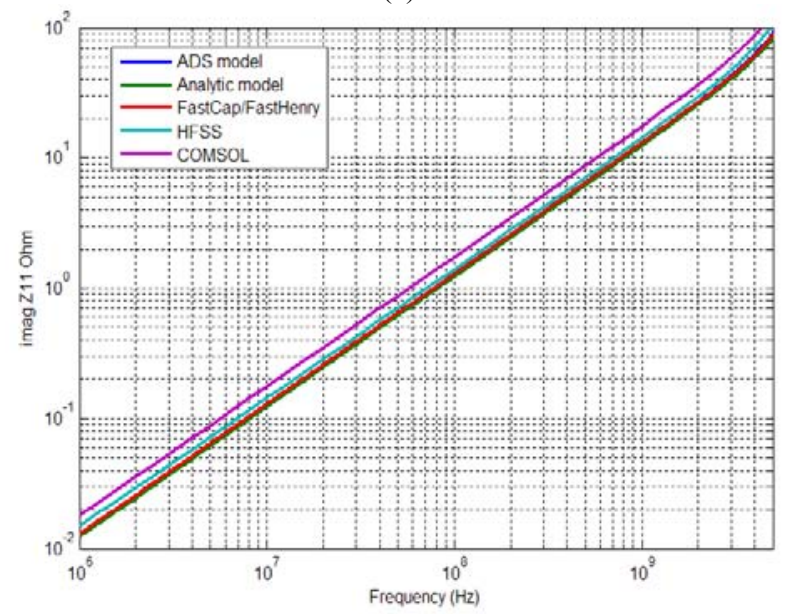

(b)

Fig. 5 (a) Microstrip: short: skin effect and (b) microstrip: short: series inductance $(Z=$ j.L.(.)).

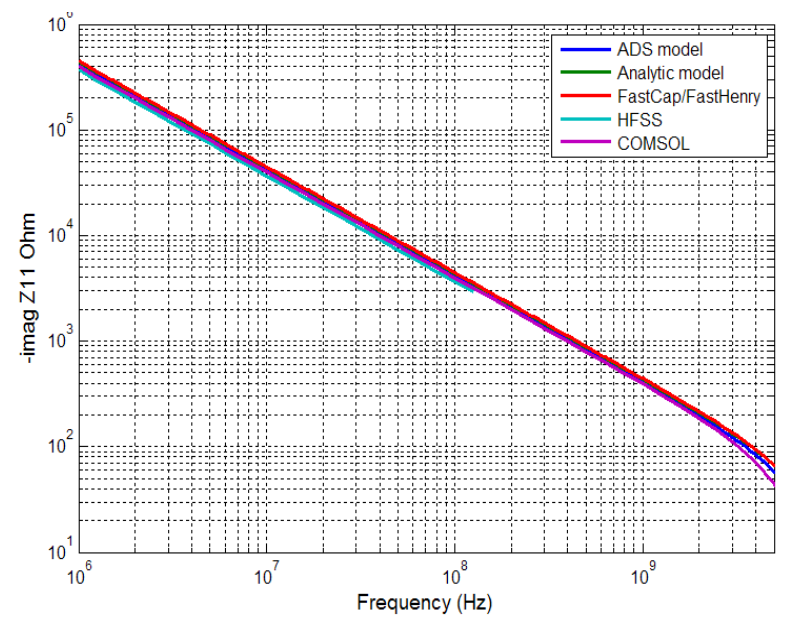

Fig. 6 Microstrip open: parallel $(Z=-j / C . \omega)$.

Fig. 7 shows a sketch of the stripline configuration. The characteristic dimensions are: 
Table 1 Summary of $\mathrm{R}, \mathrm{L}, \mathrm{C}$ results coming from some industrial simulators.

\begin{tabular}{lllll}
\hline & $\mathrm{R}_{\mathrm{S}} @ 10 \mathrm{MHz}(\mathrm{m} \Omega)$ & $\mathrm{L}_{\mathrm{S}} @ 10 \mathrm{MHz}(\mathrm{nH})$ & $\mathrm{C}_{\mathrm{P}}(\mathrm{fF})$ & $\mathrm{Z}_{0}(\Omega)$ \\
\hline Analytic & 4.7 & 1.96 & 362 & 73.6 \\
Fast henry/fast cap & 4.8 & 2.09 & 357 & 76.5 \\
ADS model & 4.6 & 2.05 & 389 & 72.6 \\
HFSS & 3.26 & 2.28 & 433 & 72.56 \\
Comsol & 5 & 2.01 & 399 & 83.9 \\
\hline & $\mathrm{RS} @ 1 \mathrm{GHz}(\mathrm{m} \Omega)$ & $\mathrm{LS} @ 1 \mathrm{GHz}(\mathrm{nH})$ & $\mathrm{CP}(\mathrm{fF})$ & $\mathrm{Z}_{0}(\Omega)$ \\
\hline Analytic & 46.8 & 1.96 & 362 & 73.6 \\
Fast henry/fast cap & 36.8 & 2.06 & 357 & 75.9 \\
ADS model & 47.4 & 2.05 & 389 & 72.6 \\
HFSS & 73.6 & 2.26 & no convergence & 72.56 \\
Comsol & 29.7 & 1.99 & 399 & 83.6 \\
\hline
\end{tabular}
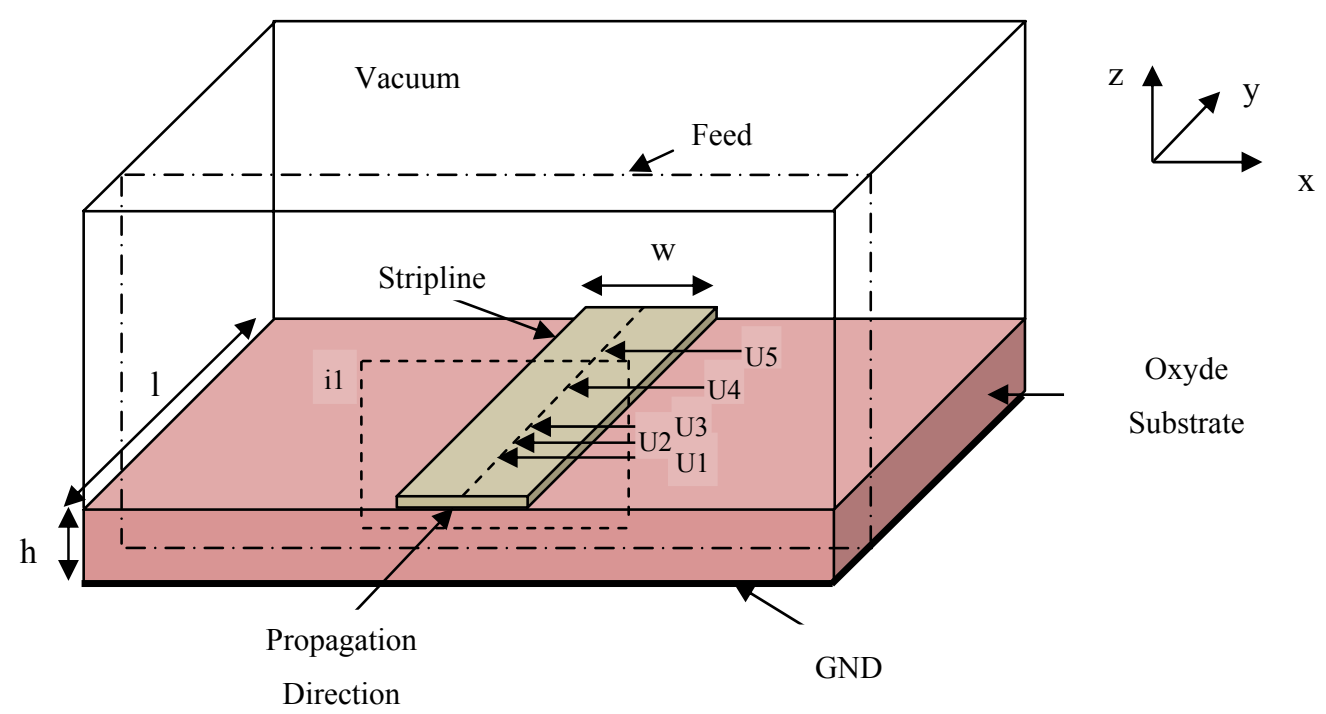

Oxyde

Substrate

Fig. 7 Geometric structure of a stripline reference.

Width: $\mathrm{w}=1 \mathrm{~mm}$;

Thickness: $\mathrm{t}=35 \mu \mathrm{m}$;

Length: $1=5 \mathrm{~mm}$;

Ground plane: $\varepsilon_{\mathrm{r}}=1.0, \sigma=45.10^{6} \Omega^{-1} \mathrm{~m}^{-1}$ and $\mu_{\mathrm{r}}=1.0$;

Substrate: oxide: $\mathrm{h}=1 \mathrm{~mm}, \varepsilon_{\mathrm{r}}=4.0, \sigma=0 \Omega^{-1} \mathrm{~m}^{-1}$ et $\mu_{\mathrm{r}}=1.0$;

Vacuum: $\varepsilon_{\mathrm{r}}=1.0, \sigma=0 \Omega^{-1} \mathrm{~m}^{-1}$ et $\mu_{\mathrm{r}}=1.0$.

The TEM field distribution map or the electric and magnetic fields are calculated inside a defined domain.

A Gaussian pulse, oriented parallel to positive $y$-axis (see Fig. 7) is injected, bridging the gap between the ground plane and the strip conductor at the desired source location (called "feed"), modulating the quiescent electromagnetic field. The probe points (sensors in Sentaurus) are called: $\mathrm{u}_{1}, \mathrm{u}_{2}, \ldots$.
The propagating Gaussian pulse (amplitude: 1 ' $\mathrm{V}$ ', standard deviation: $8.10^{-10} \mathrm{~s}$, Tpeak $=7.10^{-11} \mathrm{~s}$ ) shows deformation (Fig. 8) due to the dispersion, because of inhomogeneous materials. Working in a quasi-TEM excitation is sufficient up to tens of $\mathrm{GHz}$. We also have noted that the simulator is able to take into account the thickness of the strip conductor; the Electric field is stronger when the thickness is "zero" (strong discontinuity), especially at the corners of the strip.

Figs. 9-10 show respectively the geometry and the waves, incident and reflected, at the strip line input ( probe in " $u_{1}$ ": see Fig. 7), for an open structure (a) or a short one (b), keeping respectively the width to 1 $\mathrm{mm}$ and the thickness to $35 \mu \mathrm{m}$. For instance, we found a phase shift of $0^{\circ}$ for the "open", and $180^{\circ}$ for 


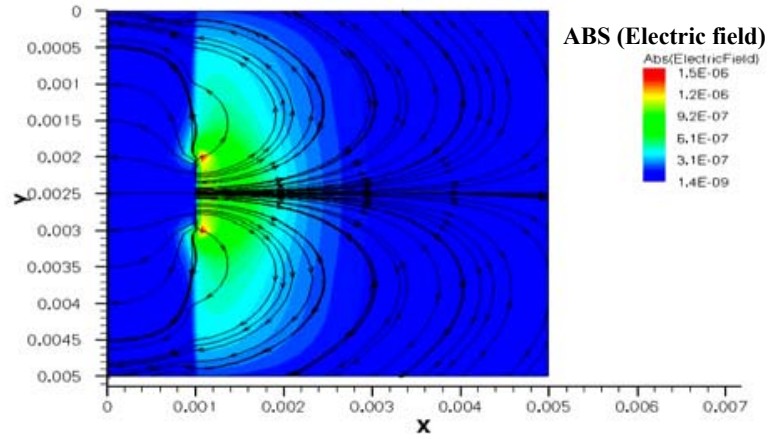

(a)

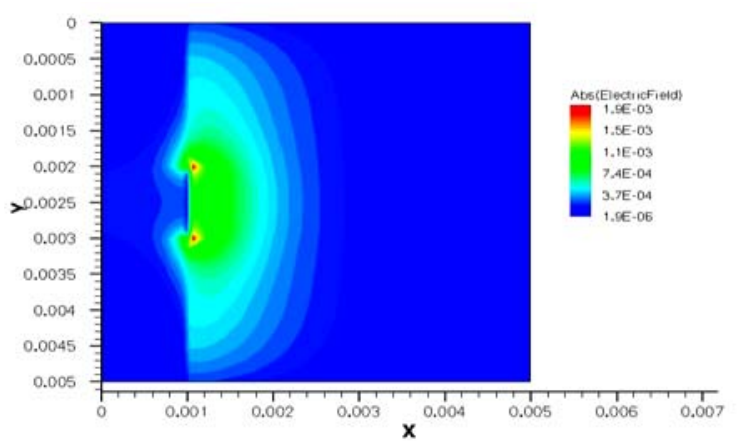

(b)

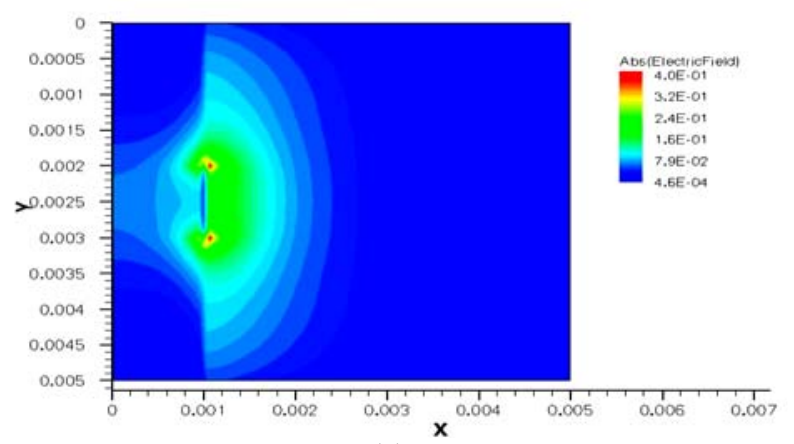

(c)

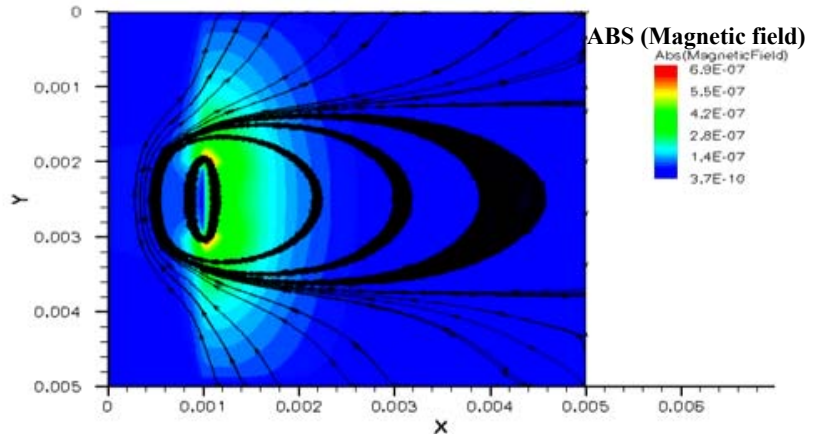

(d)

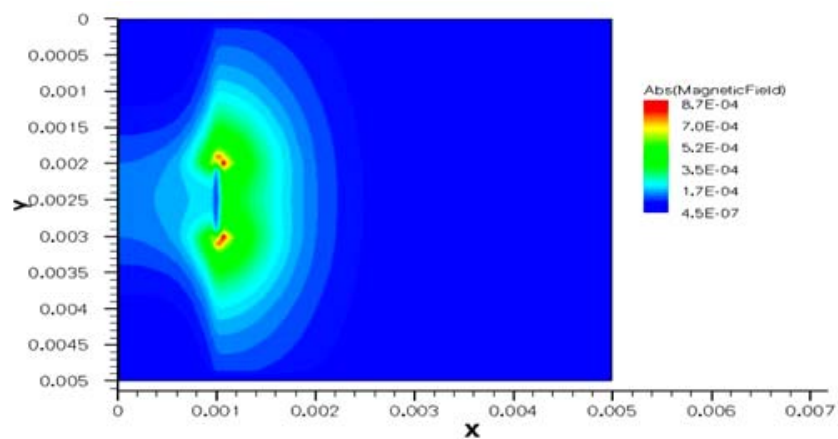

(e)

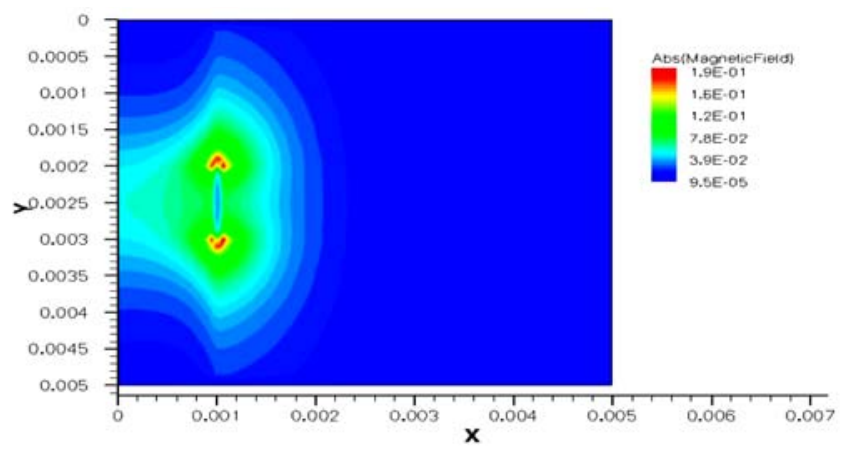

(f)

Fig. 8 Transient spatial distributions in a cross section in the middle of the stripline: (a), (b) and(c) of the electric field; (d), (e) and (f) of the magnetic field, induced by the propagation of EM wave.

the "short".

The characteristic impedance (Fig. 11) shows a known behaviour of the stripline.

Imaginary part oscillate a little bit around zero (Fig. 11a); it surely due to the $\Delta \mathrm{t} / 2$ shift between current end voltage (cf. Leapfrog method [1]).

Absorbing boundary conditions [11-12]) are also a physico-mathematical field to be investigated in-depth (it is one of the reasons we began to build our own simulator).

"Short" (Fig. 11b): we see resonances - the first one at $8 \mathrm{GHz}$ : The real part of the impedance is very near the same apart the resonances, which are not the case for the imaginary one: Its magnitude is higher below those resonances, due perhaps to an inductive effect.

The fact that the experimental (not presented here) and numerical results agree so well implies that the numerical simulation does a pretty job, picking up the parasitic capacitance or inductance (cf. oscillations) which may be present between the terminals of the device. Finally, in Fig. 12, are presented the real and the imaginary part of the $S_{11}$ parameter for a short strip line; for very low frequencies (null load) the reflected and incident waves have a $\pi$ phase gap. 


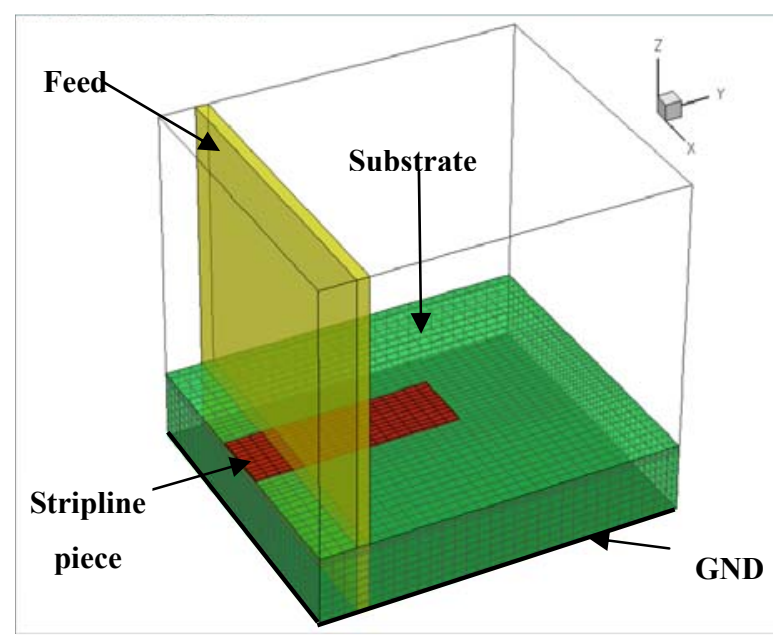

(a)

Fig. 9 (a) Open geometry and (b) short geometry.

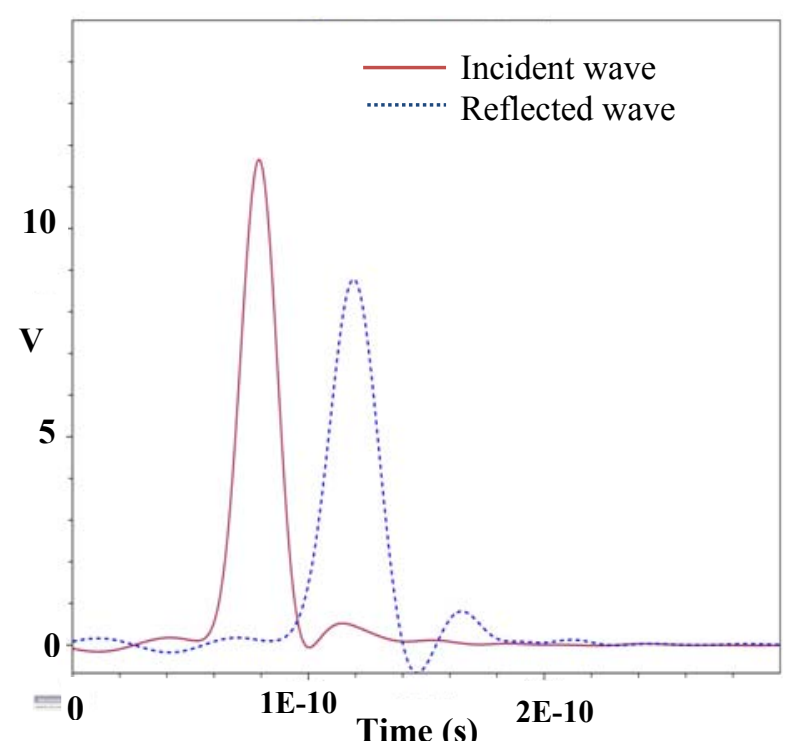

(a)

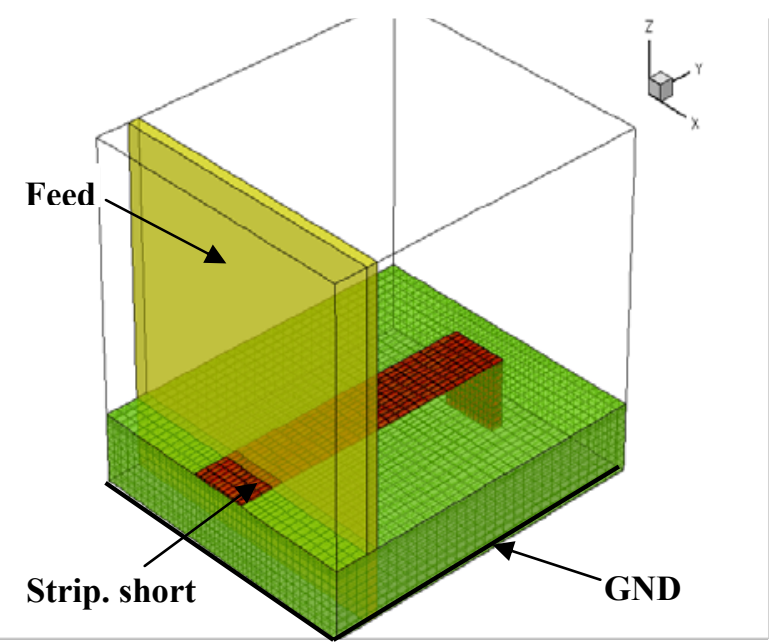

(b)

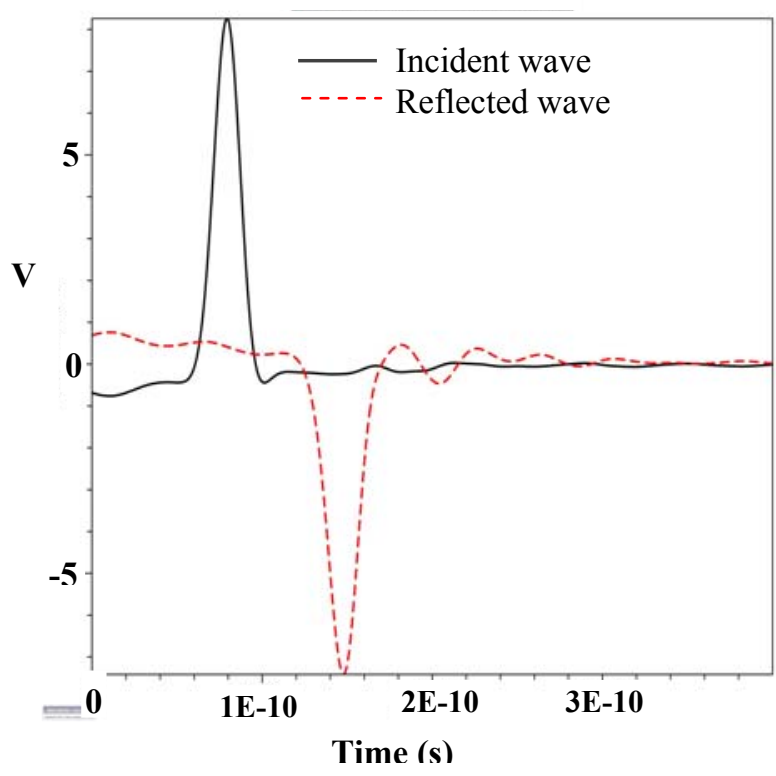

(b)

Fig. 10 (a) Stripline input and (b) stripline input.

\section{Crosstalk Attenuation with Ground Plane Structures}

To seek the efficiency of a ground plane structures in 3D integrated circuits and the robustness of the simulator, we inspect the connection system depicted in Fig. 13.

The stimulus is a smooth Gaussian pulse: amplitude: 1, standard deviation: $10^{-11} \mathrm{~s}$.

The "active area"-the target-acts as a lower device's analog transistor, the metal line ("wire"- the attacker) represents the upper device's digital interconnect lines that induce coupling; the ground plate isolates these two metals.

Presented are on Figs. 14-16 the first simulations.

Evidently, simulation results point out the size dependant shadowing of the ground plate.

An optimum seems obtained (see Fig. 16); with the medium size grounded plane, a surge appears in the attacker's voltage. The system could act as a LC-like resonator; there is some energy oscillation between 


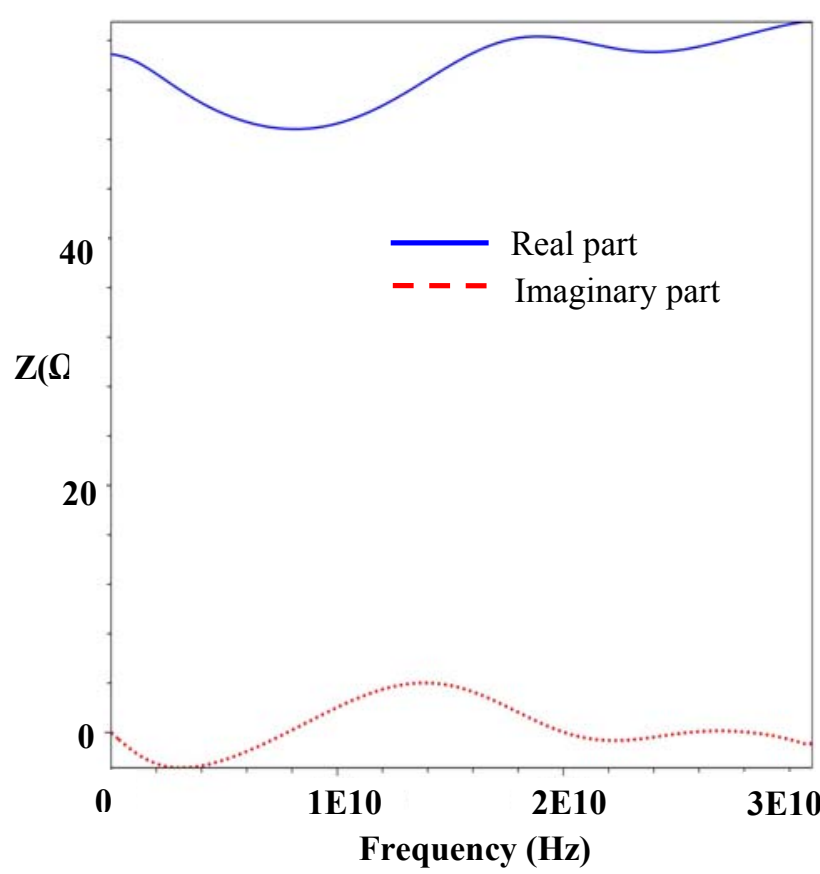

(a)

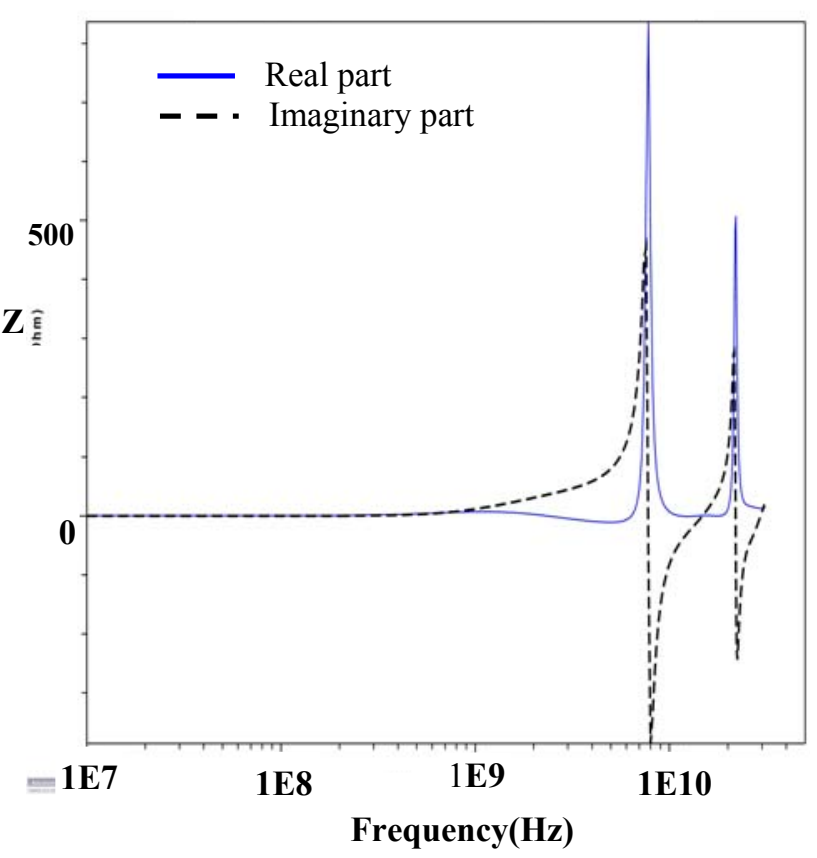

(b)

Fig. 11 (a) Open impedance and (b) short impedance.

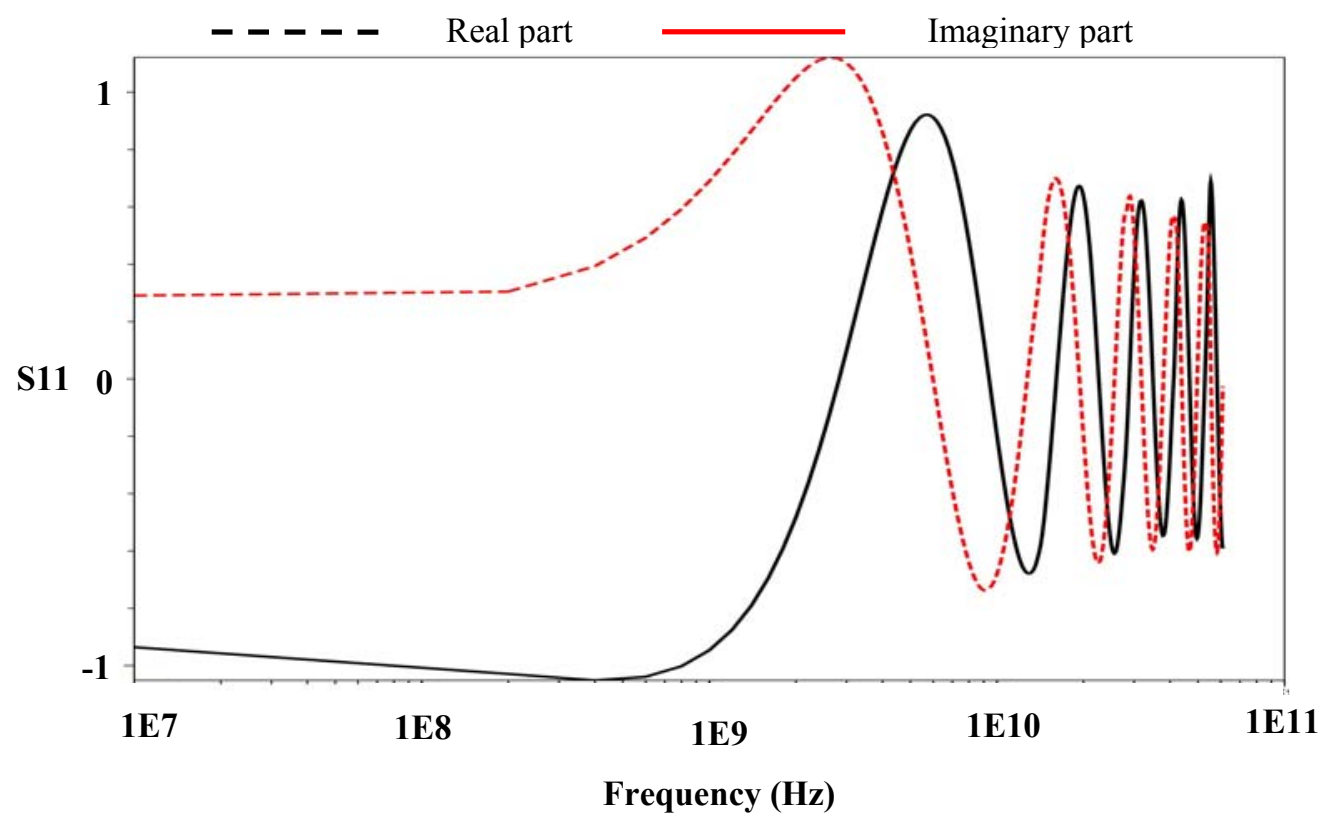

Fig. 12 Short stripline.

attacker and target: the attacker becomes alternatively the target (the target becomes the attacker). Some dimension (then frequency) dependent resonance could intervene (cf. cavities or surge in low and middle frequency RLC circuits). We confirm also the inefficiency of a floating shield.

\section{Via Electromagnetic Behaviour}

Vias are interconnects commonly found on multilayer printed or integrated circuits [13-14]. Often, they provide some path to ground or to other device via some RDL (Re-Distribution Layer). In a circuit board 


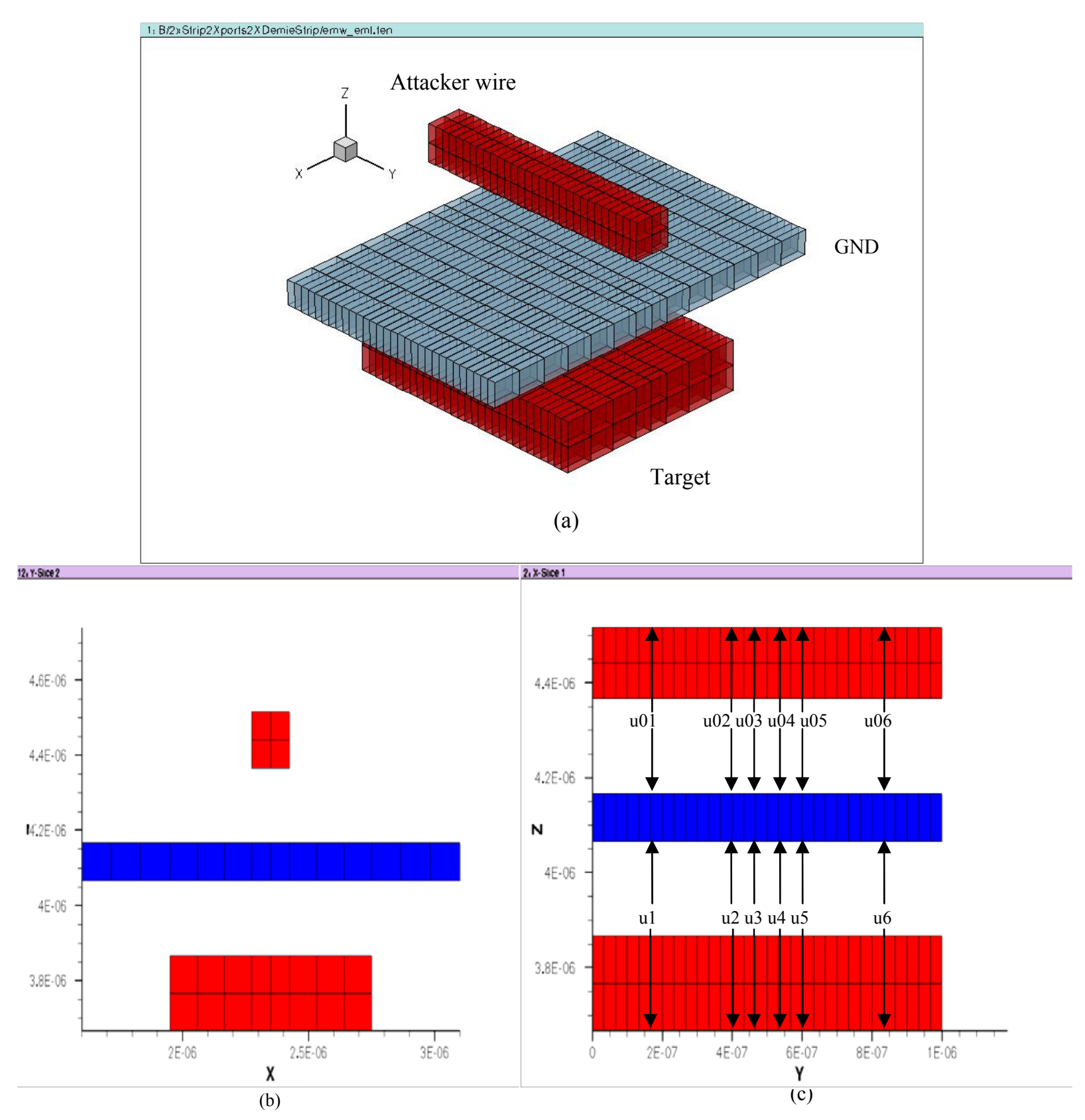

Fig. 13 Multilayered structure: (a) 3D structure; (b) cut along the $y$ axis; (c) cut along the $x$ axis.

application, some parallel conducting planes may be on a constant potential, or grounded. This has no effect on the transient time simulation since this latter is only superposed to the "quiescent point". Fig. 17 shows a $3 \mathrm{D}$ view of a circular hollow via; it is asymmetrical since the two stripline widths are different.

The characteristic dimensions are

Striplines widths: $\mathrm{w}=0.2 \mathrm{~mm}$ and $0.4 \mathrm{~mm}$;

Striplines thickness: $\mathrm{t}=50 \mu \mathrm{m}$;
Striplines length: $1=3.6 \mathrm{~mm}$;

Via height: $0.6 \mathrm{~mm}$;

Via diameter: $0.6 \mathrm{~mm}$;

Via thickness: $0.2 \mathrm{~mm}$.

The via goes to a ground plane; others shielding planes are added on the top and the bottom. Transient simulations are performed for each stripline without being connected to the via, for extraction of incident pulses, and, for instance, characteristic impedance. 

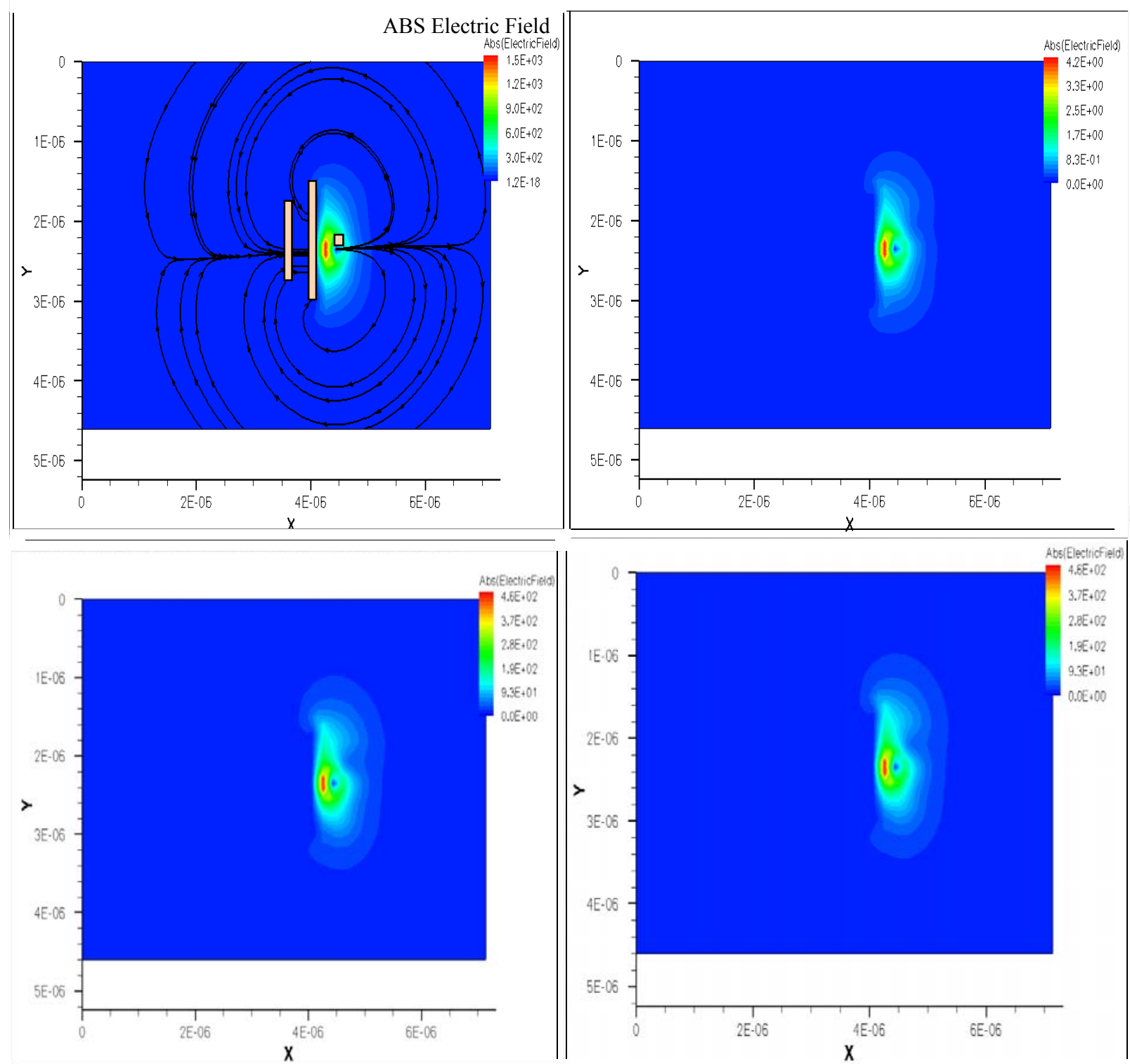

Fig. 14 Transient time spatial distributions in a $<x, z>$ plane, probe in " $u_{4}$ ", cf. Figs. 13a and 13c: (a), (b) and (c) of the electric field; (d), (e) and (f) of the magnetic field, induced by the propagation of EM wave.

Simulations for the striplines connected to the via, with each port excited, are needed for extraction of scattered pulses.

Fig. 18 presents the electric field distribution maps. We can see the propagation pace of the electric field along the structure.

The S-parameters are shown in Fig. 19.

At low frequency, all the signal is transmitted through the via, since in high frequency appear oscillations that we suppose coming from inductive and capacitive phenomena.
This simulator permit also to take into account ground bounces induced by some parasitic electro-magnetic waves.

Fig. 20 shows voltages observed at the two ports, for the transient regime. The pulse is quite well transmitted to the stripline. We observe also important oscillations.

Our further work will be to extract, not only the R.C passive equivalent parameters, but also the inductances, which are not so easy to grasp (not only because of the skin effect). 


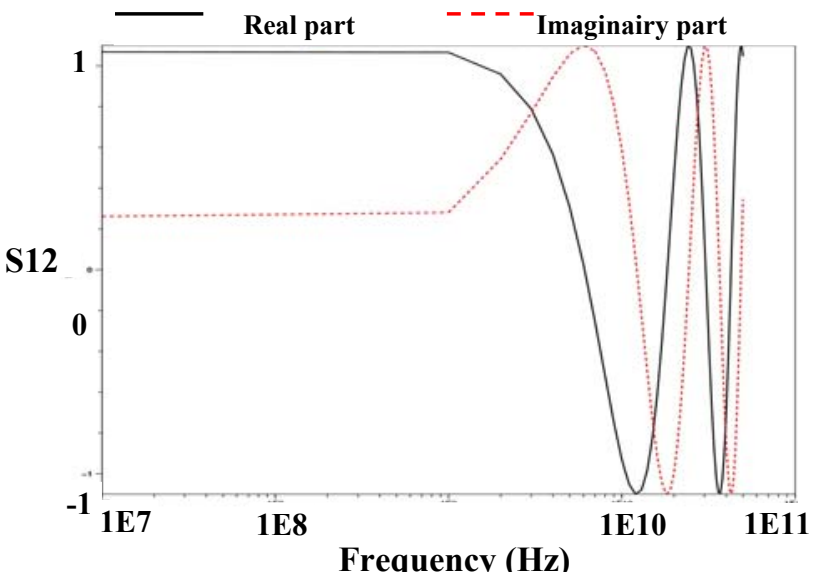

(a)

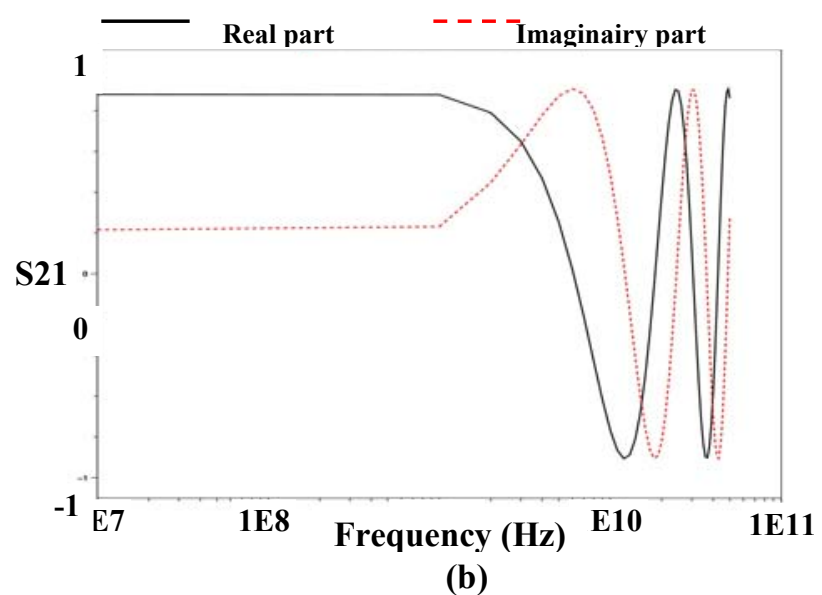

(b)

Fig. 15 Transmission parameters of the multi-layered structure (cf. Fig. 13a).

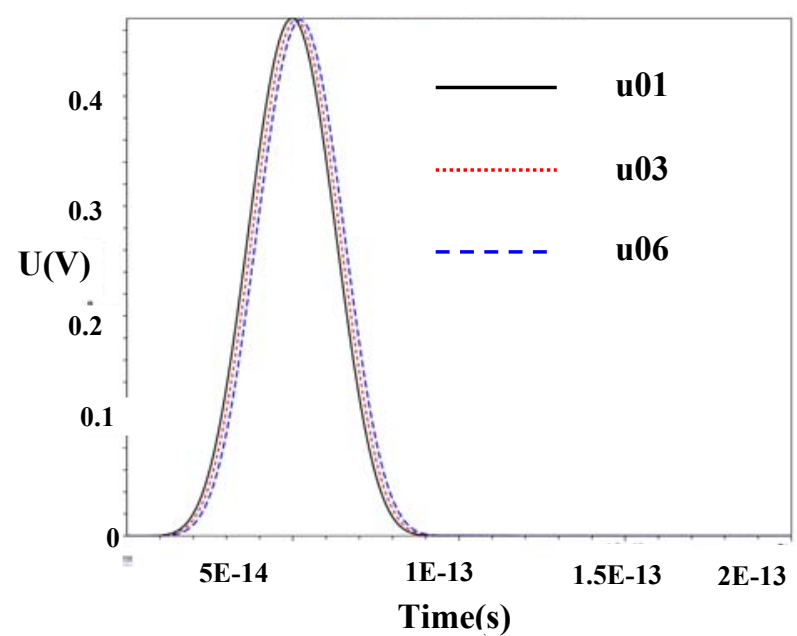

(a)

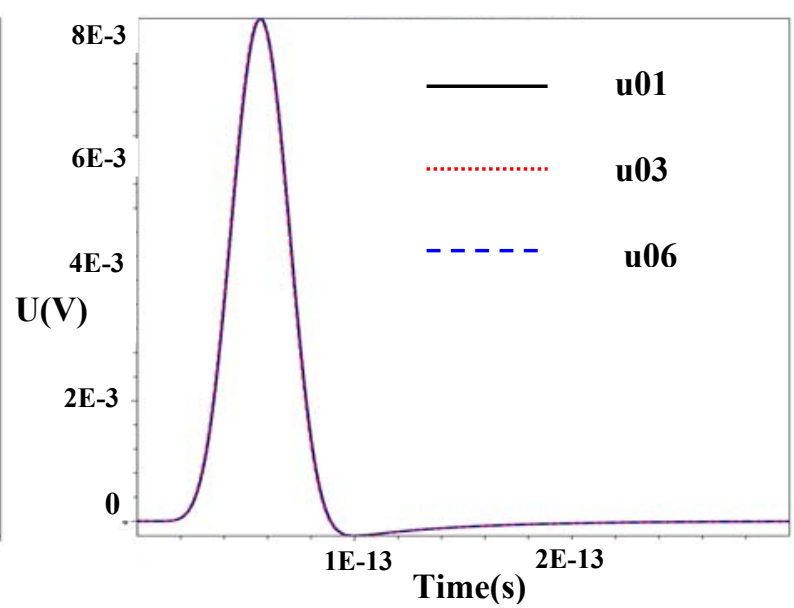

(b)

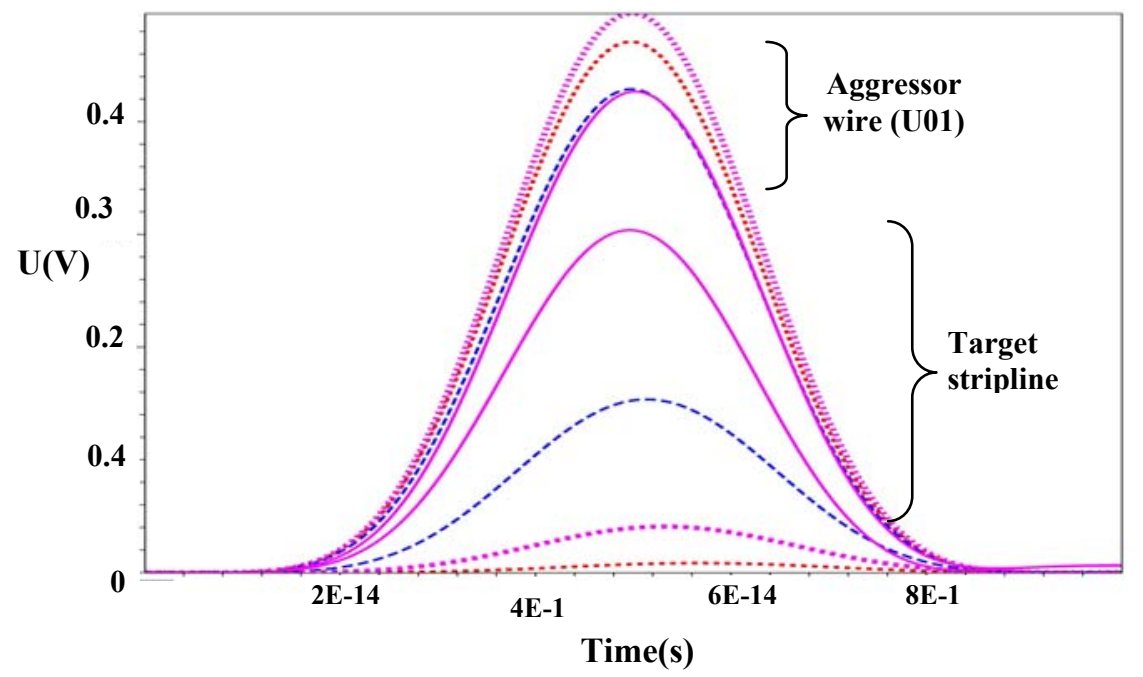

(c)

Fig. 16 Pulse wave on (a) the attacker, (b) the target and (c) at different points (cf. Fig. 13c); pulse taken near the input, for different shield surfaces (shield grounded: $1.5 \times 1 \mu \mathrm{m}^{2}: \ldots . . . ; 0.8 \times 1 \mu \mathrm{m}^{2}:$ : ,", $; 0.15 \times 1 \mu \mathrm{m}^{2}:$----- . shield floating: $1.5 \times 1 \mu \mathrm{m}^{2}:-$ ). 


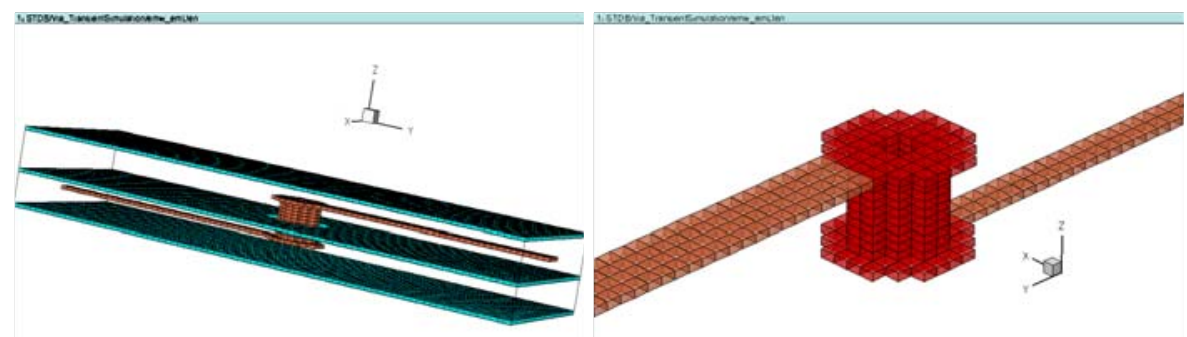

(a)

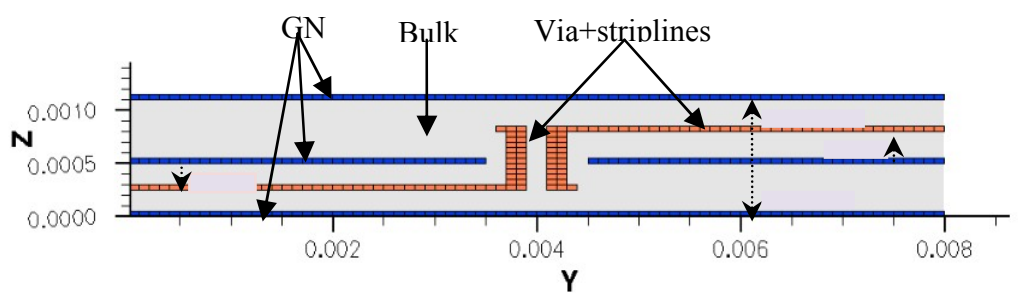

(b)

Fig. 17 Via geometry: (a) 3D Structure and (b) transversal cut in the plane $<z, y>$.
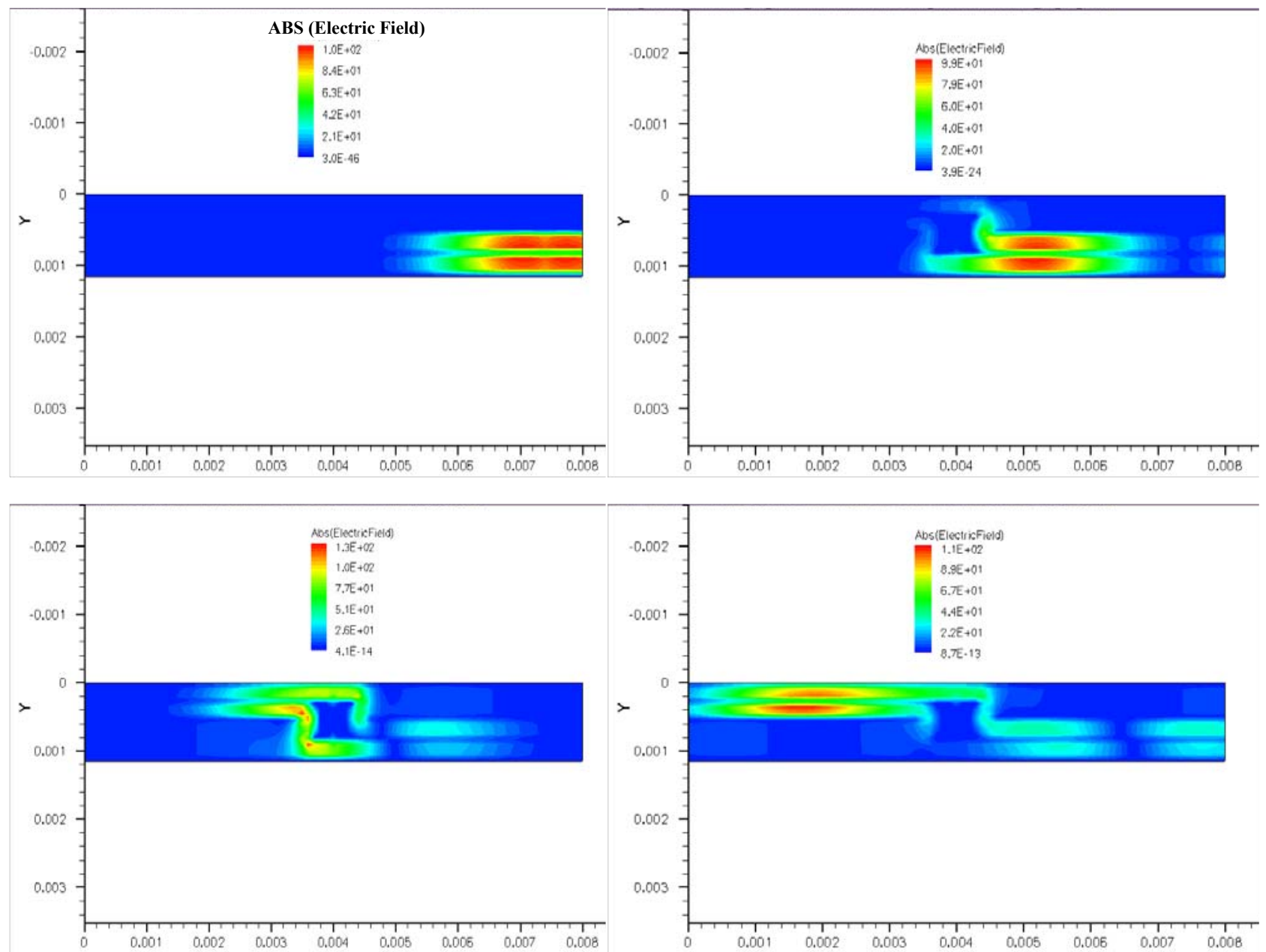

Fig. 18 Via cross section: spatial distribution of the electric field, taken at different times; wave propagation: from the right to the left. 


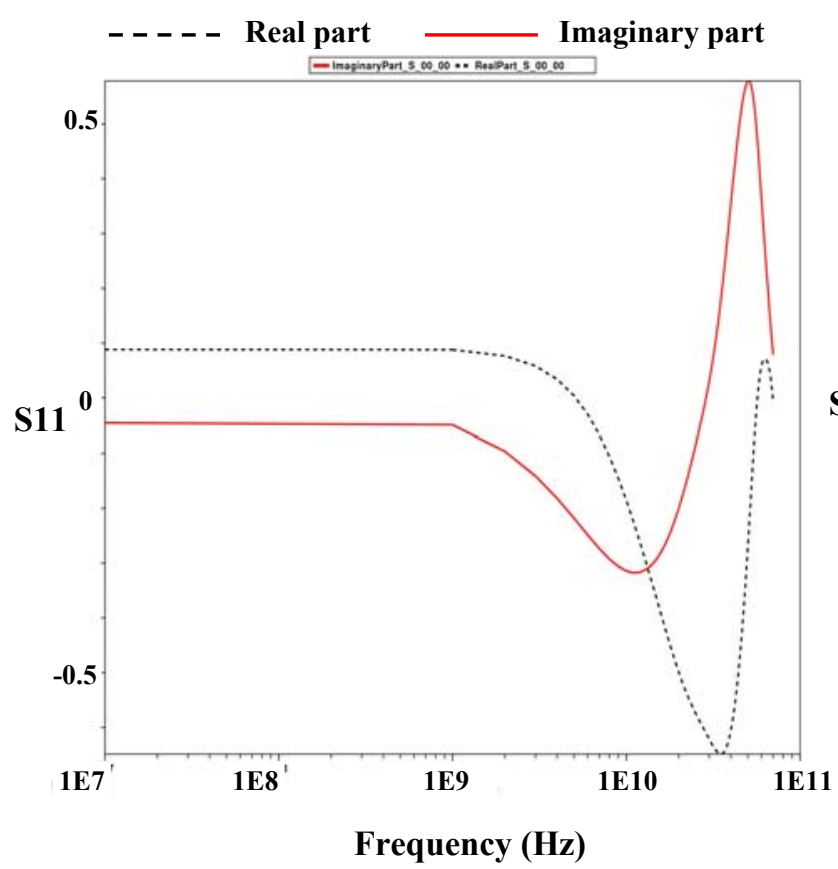

(a)

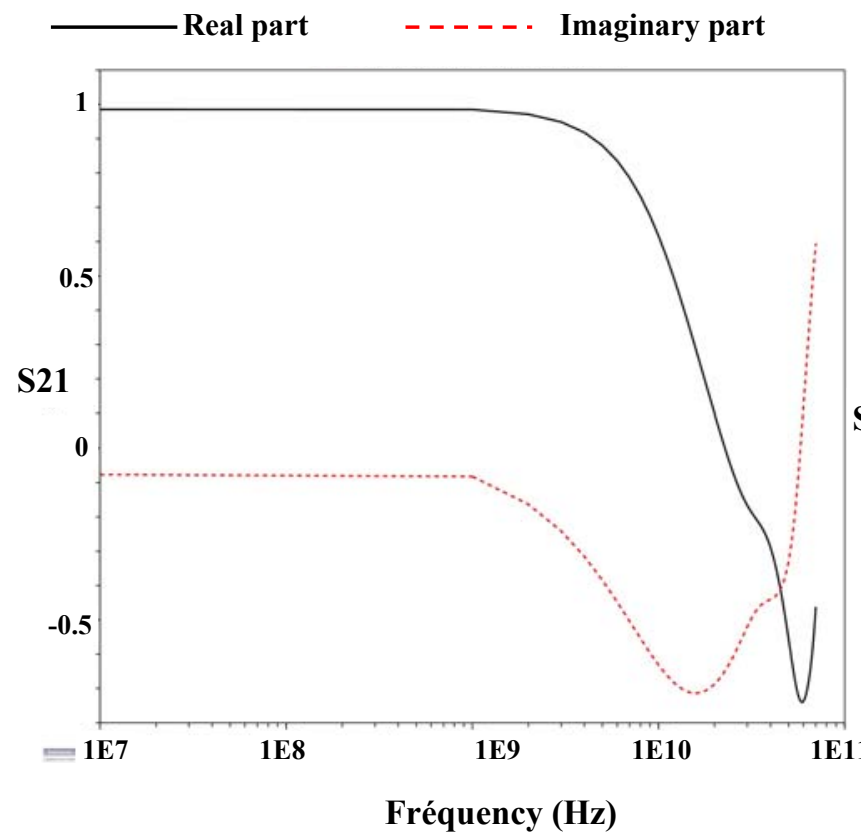

(c)

Fig. 19 Dispersion parameters of the asymmetrical via.

\section{Conclusions}

In this paper, after presenting a resolution of the curls of the electric and magnetic field, using a FDTD method (finite-difference time-domain), applied to a single conducting plate, we go on by studying the capabilities of a well-known but rarely used numerical simulator
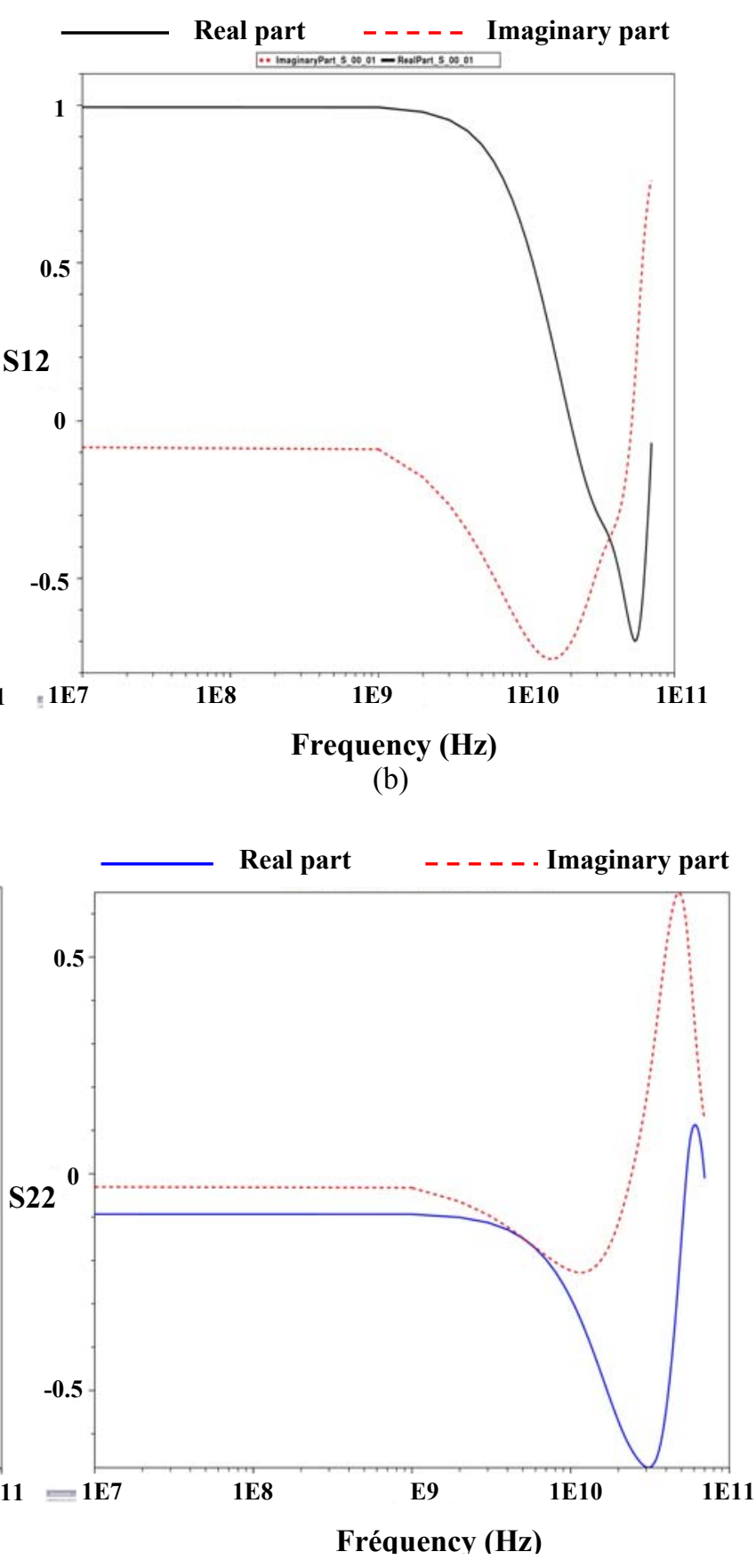

(d)

(Sentaurus) to the analysis of two or three-dimensional interconnect structures, developed on time domain; in this case, FFTs are taken as post processing.

We did go on to the crosstalk study between two striplines shielded by some ground plane, and we show that an optimum surface of the shield should be achieved. 


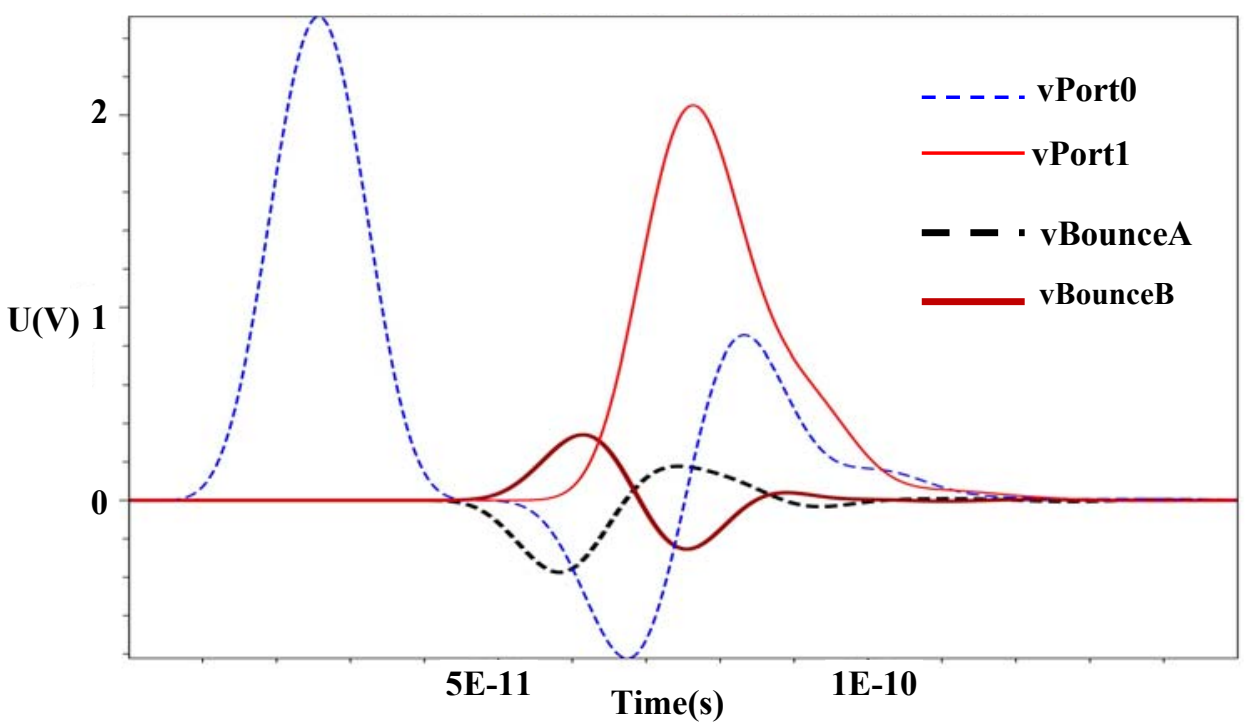

Fig. 20 Transient potentials: pulse applied on le Port0 ( see Fig. 17b).

Our conclusion is that, for the following works, we essentially will use the "FDTD" simulator for two essential reasons. The first one: This simulator is also dedicated to device analysis. The other reason is we will go on to develop our own time domain simulator, taking care of the no totally resolved boundary problems, nowadays.

\section{Acknowledgment}

This work is supported by the Cluster for Application and Technology Research in Europe on Nano-Electronics (CATRENE): 3D-TSV Integration for Multi-Media and Mobile applications (3DIM $\left.{ }^{3}\right)$.

\section{References}

[1] K.S. Yee, Numerical solution of initial boundary value problems involving Maxwell's equations in isotropic media, IEEE Transactions on Antennas and Propagation AP-14 (1966) 302-307.

[2] Sentaurus Device Electromagnetic Wave Solver User Guide (Version A-2008.09, September 2008 SYNOPSYS).

[3] W. Fichtner, D. Rose, R. Bank, Semiconductor device simulation, IEEE Trans. on Elec. Dev. 4 (1983) 1018-1030.

[4] A. Taflove, Basis and application of finite-difference time domain (FDTD) techniques for modelling electromagnetic wave interactions (short course notes), in: 1992 IEEE Antennas Propagat. Soc. In. Symp. and URSI 21 Radio Sci. Meeting, Chicago, IL, July, 1992.
[5] Agilent, Advanced Design System, US: Agilent Tech., available online at: http://eesof.tm.agilent.com.

[6] M. Kamon, M. Tsuk, J. White, Fast-henry: a multipole-accelerated 3-D inductance extraction program, in: Proceedings of the 30th international Design Automation Conference Dallas, Texas, United States, 1993, pp. 678-683.

[7] K. Nabors, J. White, Fastcap: a multipole accelerated 3D extraction program, IEEE Transactions on Computer-Aided Design of Integrated Circuits and Systems, 1991.

[8] HFSS-Free Trial: 3D Full-Wave Electroma-Gnetic Field Simulation.

[9] COMSOL, Multiphysics Modeling and Simulation, available online at: http://www.comsol.fr/.

[10] G.E. Ponchak, RF transmission lines on silicon substrates, in: Proceeding of GAAS1999, Munich, 1999.

[11] V. Joolen, B. Neta, D. Givoli, High order higdon-like boundary conditions for exterior transient wave problems, International Journal for Numerical Methods in Engineering 63 (2005) 1041-1068.

[12] G. Mur, Absorbing boundary conditions for the finite difference approximation of the time domain electromagnetic field, Electromagnetic Compatibility, IEEE Transactions on Electromagnetic Compatibility EMC-23 (1981) 377-382.

[13] L. Cadix , C. Bermond, C. Fuchs, A. Farcy, P. Leduc, L. DiCioccio, et al., RF characterization and modelling of high density through silicon vias for 3D chip stacking, Micrelectronic Engineering 87 (2010) 491-495.

[14] I. Savidis, S. Alam, A. ScottPozder, R. Jones, R. Chatterjee, Electrical Modeling and Characterization of Through-Silicon Vias (TSVs) for 3D Integrated Circuits, available online at: www.elsevier.com/locate /mejo. 